\title{
Spontaneous recovery from isolated post-traumatic central hypogonadism in a woman
}

\author{
Rosaria M. Ruggeri ${ }^{1}$, Giovanni Smedile ${ }^{1}$, Francesca Granata ${ }^{2}$, Marcello Longo ${ }^{2}$, \\ Salvatore Cannavò ${ }^{1}$, Nicholas J. Sarlis ${ }^{3}$, Francesco Trimarchi ${ }^{1}$, Salvatore Benvenga ${ }^{1,4}$
}

${ }^{1}$ Clinical Experimental Department of Medicine and Pharmacology, Section of Endocrinology, ${ }^{2}$ Department of Radiolo gical Science, University of Messina, Messina, Italy ${ }^{3}$ Department of Medical Affairs, Oncology - US, Sanofi-Aventis US, Bridgewater, NJ, USA, 'Master of Childhood, Adolescent and Women's Endocrine Health, University of Messina, Italy

\begin{abstract}
OBJECTIVE. Hypopituitarism is a recognized complication of Traumatic Brain Injury (TBI). Resolution of established anterior pituitary hormones deficiency is rare. CASE REPORT. A woman was initially presented at the age of 22 years with amenorrhoea. Two years earlier she had been involved in a car accident with consequent TBI. At our evaluation, serum oestradiol $\left(E_{2}\right)$ was low (3 pmol/l), Luteinizing Hormone (LH) and Follicle Stimulating Hormone (FSH) were borderline low [1.7 and $2.5 \mathrm{mIU} / \mathrm{l}$, normal range (NR) for 20-35 yr old women being 1.413 and 2.5-13, respectively] with poor response to Gonadotropin Releasing Hormone (GnRH) $(\Delta \mathrm{LH}=2.4$ and, $\Delta F S H=4.0 \mathrm{mIU} / \mathrm{l})$, while serum Prolactin $(P R L)$ was elevated $(951 \mathrm{mIU} / \mathrm{l}$; NR: 102-496). No other pituitary hormone deficiencies were observed. Magnetic resonance imaging (MRI) showed a partially empty sella and very thin stalk. Ten years later a new endocrine evaluation was performed. On day 4 of her menstrual cycle, serum values of PRL $(196 \mathrm{mIU} / \mathrm{l})$, FSH $(4.7 \mathrm{mIU} / \mathrm{l}), \mathrm{LH}(4.8 \mathrm{mIU} / \mathrm{l})$ and $\mathrm{E} 2(103 \mathrm{pmol} / \mathrm{l})$ were within normal limits for women aged 20-35. Six months after this evaluation (that is, 12 years after trauma), the patient became pregnant and delivered a healthy baby. CONCLUSIONS. Our case shows that spontaneous resolution of post-head trauma anterior hypopituitarism occur even many years after head injury. Medicolegal implications are self-evident.
\end{abstract}

Key words: Gonadotropin deficiency, Infertility, Oligo-amenorrhoea, Post-head trauma hypopituitarism,

Address for correspondence:

Rosaria M. Ruggeri, MD, Unità di Endocrinologia,

Dipartimento Clinico-Sperimentale di Medicina e

Farmacologia, Padiglione H, 4 piano, Policlinico Universitario

"G. Martino", 98125 Messina, Italy, Tel.: +39-090-2213840,

Fax: +39-090-2213517, E-mail: rmruggeri@unime.it

Received 25-01-10, Revised 22-06-10, Accepted 20-07-10

\section{INTRODUCTION}

Brain injury such as head trauma [Traumatic Brain Injury (TBI) or Subarachnoid Hemorrhage (SAH)] represent one of the leading causes of pituitary insufficiency. ${ }^{1-4}$ The risk of post-head trauma hypopituitarism is much greater than previously suspected and, 
given the increasing incidence of TBI, particularly after car accidents, this constitutes a very relevant clinical problem.

In the year 2000 , a review on post-head trauma hypopituitarism included 367 cases of isolated or multiple deficiency of anterior pituitary hormones subsequent to TBI. ${ }^{1}$ The interest triggered by the review, which concluded that post-traumatic hypopituitarism was indeed less rare than commonly considered, was such that a total of 192 cases were reported from the year 2000 through April, 2005. ${ }^{5}$ In the year 2007 a metanalysis by Schneider et al identified 19 studies on hypopituitarism following TBI or SAH, which included 1137 patients. ${ }^{6}$ The prevalence of endocrine dysfunction following TBI ranged from $15 \%$ to $68 \%$, and from $37.5 \%$ to $55 \%$ following SAH. ${ }^{6}$ There was, however, considerable disagreement on the relative frequency of the various anterior pituitary axes affected. SAH was associated with significantly higher frequencies of pan-hypopituitarism, Growth Hormone (GH) deficiency and Adrenocorticotrophin (ACTH) deficiency than TBI. After TBI, deficiencies of Luteinizing Hormone (LH)/follicle-stimulating hormone (FSH) and $\mathrm{GH}$ were significantly more common than ACTH deficiency and thyroid-stimulating hormone (TSH) deficiency. ${ }^{6}$ In particular, a prospective 12month study by Aimaretti et al on 102 patients who had TBI or SAH found that hypopituitarism occurred in about $1 / 3$ of patients and that the prevalence of secondary hypogonadism was $11.4 \% .^{7}$ A survey by Herrmann et al estimated that hypopituitarism occurs in one fourth of patients recovering from severe TBI, with gonadotrophin and GH deficiency being the predominant failures. ${ }^{8}$ A more recent study by the same group confirmed the magnitude of the rate of impaired pituitary function ( $21 \%$ of TBI patients). ${ }^{9}$ The proportions of total, multiple or isolated deficit were $1 \%, 2 \%$ and $18 \%$, respectively, with secondary hypogonadism and secondary hypothyroidism being the predominant deficits. ${ }^{9}$ Another 12-month prospective study by Klose et al found that early post-traumatic hormone alterations, including gonadotrophin deficiency or central hypothyroidism, were present in 35 of the 46 (76\%) TBI patients studied. These deficits persisted at 12 months in 5/46 (10.8\%) subjects, predominantly with GH deficit, and they were correlated with the severity of TBI. ${ }^{10}$
Finally, hyperprolactinaemia has also been reported in addition to isolated or combined hormonal deficits. In the review by Benvenga et al hyperprolactinaemia was reported in up to $48 \%$ of patients. ${ }^{1}$ Aimaretti and coworkers demonstrated mild hyperprolactinaemia in 3/70 (4.2\%) of TBI patients, which was associated with pituitary deficits in two of the three. The 12-month retesting demonstrated persistent mild hyperprolactinaemia in 4/70 (5.7\%) TBI patients, which was not associated with severe pituitary deficits. ${ }^{7}$ In the prospective study by Tanriverdi et al, six of the 50 subjects studied $(12 \%)$ had hyperprolactinaemia in the acute phase and PRL levels were correlated negatively with the Glasgow Coma Scale (GCS). Mild hyperprolactinaemia was still present in four men (7.8\% of patients) at the 12 -month evaluation. ${ }^{11}$ In a similar study by Schneider et al, PRL was elevated in nine out of 77 TBI patients (11.6\%), and hyperprolactinaemia was still present in $6 / 9$ at 12 months..$^{12}$ In all the reported cases, hyperprolactinaemia had no clinical relevance, so that no treatment with dopamineagonists had been applied.

Since post-head trauma hypopituitarism is obviously not a rare disease, there is a consensus that patients who suffer head trauma should undergo periodic endocrine evaluation. ${ }^{13}$ Otherwise, pituitary failure may be overlooked due to its subtle clinical manifestations and may contribute to the physical and psychological sequelae of brain trauma. The present case demonstrates that spontaneous resolution of a post-traumatic anterior hypopituitarism may occur even many years after the head injury.

\section{PATIENT'S DESCRIPTION}

We present a 22-yr old woman referred to our Endocrinology Clinic for evaluation of amenorrhoea and infertility (the latter being diagnosed a propos of the patient's inability to conceive despite more than one year of regular intercourse without contraception). Two years earlier she had been involved in a car accident with consequent multiple traumas, including TBI. Her subsequent 4-week long hospitalization had been complicated by transient diabetes insipidus. After TBI and hospitalization, the patient had amenorrhoea, whereas prior to the accident her menstrual cycle had been regular. 
On physical examination the patient's weight was $76 \mathrm{~kg}$ and her height $168 \mathrm{~cm}\left(\right.$ BMI $\left.27 \mathrm{~kg} / \mathrm{m}^{2}\right)$. Her blood pressure was $120 / 80 \mathrm{mmHg}$ and her pulse rate was $64 / \mathrm{min}$. Galactorrhoea was absent.

Pertinent hormonal data are presented in Table 1. At our first evaluation, serum oestradiol $\left(\mathrm{E}_{2}\right)$ was low (3 pmol/L), while LH and FSH were borderline low (1.7 and $2.5 \mathrm{IU} / \mathrm{L}$ ) and poorly responsive to $100 \mu \mathrm{g}$ iv $\mathrm{GnRH}$ (peak at 30 min with $\Delta \mathrm{LH}=2.4$ and $\Delta \mathrm{FSH}=4.0$ IU/L). Serum PRL was elevated (951 mIU/L), but responsive to Thyroid Stimulating Hormone (TRH) stimulus (data not shown). Serum FT4 and FT3 were normal (11.5 pmol/L and $5.9 \mathrm{pmol} / \mathrm{L}$, respectively), as were basal and TRH-stimulated TSH $(1.7 \mathrm{mIU} / \mathrm{L}$; $\Delta \mathrm{TSH}=9.7 \mathrm{mIU} / \mathrm{L})$. The $\mathrm{IGF}-1$ was $31 \mathrm{nmol} / \mathrm{L}$ and morning serum cortisol and ACTH were $321 \mathrm{nmol} / \mathrm{L}$ and $7.3 \mathrm{pmol} / \mathrm{L}$, respectively (Table 1 ).

Magnetic resonance imaging (MRI) showed a partially empty sella, a very thin stalk and a small pituitary (Figure 1). The patient refused further dynamic hormonal tests to adequately study the adrenal-axis and the $\mathrm{GH}$-axis and indeed did not even return to collect our discharge summary report with her final diagnosis or her prescriptions.

The patient returned to us ten years later. During this decade she had taken intermittently oral oestroprogestins prescribed by her gynaecologist. Discontinuation of the oestroprogestins was always followed by amenorrhoea. Of note, again the patient had tried to become pregnant but unsuccessfully. No dopaminergic agonists were prescribed for hyperprolactinaemia. Spontaneous and provoked galactorrhoea were always absent, as they had been at our initial evaluation. Because of hyperprolactinaemia, we inquired carefully about medications and diseases known to elevate circulating PRL, but none were reported.

When the patient returned to our clinic (12 years after TBI), she had discontinued oestroprogestins during the preceding six months. Unlike previous

Table 1. Data of the endocrine evaluation during the patient's first and last evaluation, i.e. 2 years and 12 years after initial head trauma.

\begin{tabular}{|c|c|c|}
\hline \multirow[b]{2}{*}{ Hormone values * } & \multicolumn{2}{|c|}{ Time after head trauma } \\
\hline & 2 years & 12 years \\
\hline \multicolumn{3}{|l|}{ FSH, IU/l } \\
\hline Baseline (2.5-13) & 2.5 & 4.7 \\
\hline GnRH-stimulated increment $(\Delta \mathrm{FSH}=5)$ & 4.0 & ND \\
\hline \multicolumn{3}{|l|}{$\mathrm{LH}, \mathrm{IU} / \mathrm{l}$} \\
\hline Baseline (1.4-13) & 1.7 & 4.8 \\
\hline GnRH-stimulated increment $(\Delta \mathrm{LH}=20)$ & 2.4 & ND \\
\hline $\mathrm{E} 2, \mathrm{pmol} / \mathrm{l}(22-609)$ & 3 & 103 \\
\hline \multicolumn{3}{|l|}{ PRL, mIU/l } \\
\hline Baseline (102-496) & 951 & 196 \\
\hline TRH-stimulated PRL increment $(\Delta \mathrm{TSH}>2$ fold $)$ & 2.1 & ND \\
\hline \multicolumn{3}{|l|}{ TSH, mIU/l } \\
\hline Baseline (0.4-4.0) & 1.7 & 1.5 \\
\hline TRH-stimulated increment $(\Delta \mathrm{TSH}=5.5)$ & 9.7 & ND \\
\hline FT4, pmol/l (9-20) & 11.5 & 15.60 \\
\hline FT3, pmol/l (2.76 - 6.45) & 5.9 & 4.0 \\
\hline Morning ACTH, pmol/l (2-11) & 7.3 & 3.32 \\
\hline Morning cortisol, nmol/1 (138-690) & 321 & 434 \\
\hline IGF-1, nmol/l (22.2-39.6) & 31 & 27.12 \\
\hline
\end{tabular}

*Normal ranges are given in parentheses. Normal range of IGF-1 is age and gender-normalised.

ND: Not Done, GnRH: Gonadotropin Releasing Hormone, FSH: Follicle Stimulating Hormone, LH: Luteinizing Hormone, $\mathrm{E}_{2}$ : Estradiol, TRH: Thyroid Stimulating Hormone, $\mathrm{FT}_{4}$ : Free Thyroxin, PRL: Prolactin, ACTH: Corticotropin. 


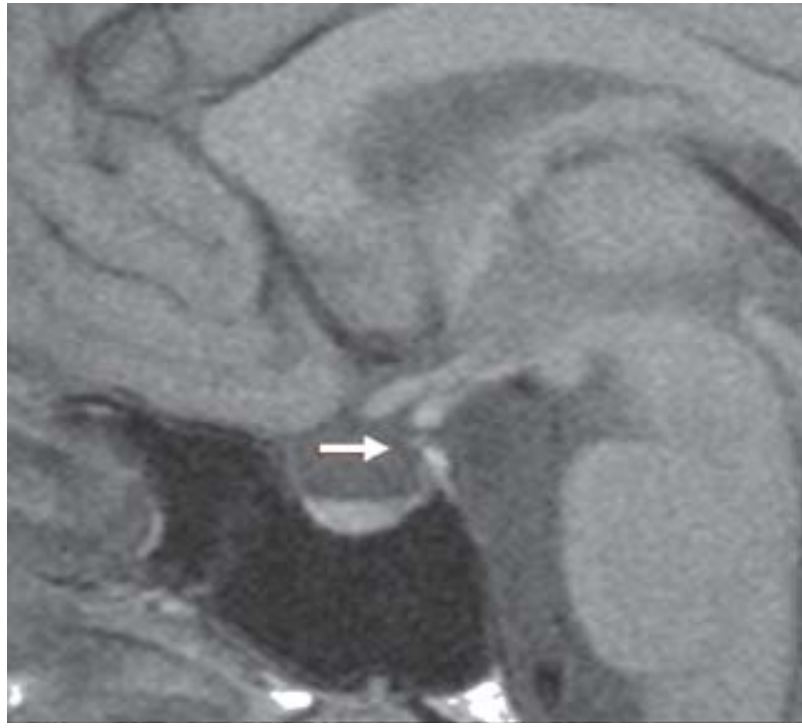

Figure 1. Sagittal T1-weighted magnetic resonance imaging showing a partially empty sella, a thin pituitary stalk (arrow) and a small pituitary. No alteration of the signal is given by the pituitary gland, indicating absence of focal abnormalities.

times, this last withdrawal of oestroprogestins was followed by spontaneous menstrual cyclicity. A new biochemical evaluation on day 4 of her menstrual cycle showed normal values of serum FT3 (4.97 pmol/L), FT4 (15.60 pmol/L), TSH (1.5 mIU/L), IGF-1 (27.2 $\mathrm{nmol} / \mathrm{L})$, cortisol $(434 \mathrm{nmol} / \mathrm{L}), \mathrm{ACTH}(3.32 \mathrm{pmol} / \mathrm{L})$, PRL (196 mIU/L), FSH (4.7 mIU/L), LH (4.8 mIU/L) and E2 (103 pmol/L). Indeed, spontaneous recovery of the gonadal function was proven by the fact that six months after this second evaluation the patient became pregnant and delivered a healthy baby.

\section{DISCUSSION}

Hypogonadotrophic hypogonadism represents the second most frequent pituitary deficit after TBI. One study on brain-injured patients found central hypogonadism in approximately $14 \%$ of patients evaluated after three months and approximately $7 \%$ of patients evaluated after 12 months. ${ }^{7}$ Similarly, Schneider et al estimated that $32 \%$ of their TBI patients had impairment of the gonadotropic axis at 3 months, which was still present in $21 \%$ of patients at 12 months. ${ }^{12}$ In another study, the two rates were $76 \%$ and $2 \%$ of TBI patients, respectively. ${ }^{10}$ In the study by Tanriverdi et al, the percentage of patients who had gonadotropin deficiency was $41.6 \%$ within $24 \mathrm{~h}$ of admission to the neurosurgery unit, but only $7.7 \%$ at the 12 months follow-up. ${ }^{11}$ It may thus be deduced that secondary hypogonadism is frequently transient during the first year after TBI. This relatively short period of transiency suggests that hypogonadism could be the result of a functional rather than an anatomic damage, perhaps reflecting a stressful condition. ${ }^{7,10,14-16}$ Accordingly, it is typically suggested that practitioners wait for the initiation of long-term hormonal replacement for gonadal axis deficits (as well as of other pituitary deficits) when such deficits appear shortly (within three months) after TBI or SAH. ${ }^{2,711,12}$

On the other hand, when pituitary hormone deficiencies are confirmed at 12 or more months after TBI, they are considered generally stable over time and, therefore, appropriate long-term replacement therapy is initiated..$^{2,7,13}$

We favour an organic nature, over a dysfunctional, stress-related nature, ${ }^{17,18}$ of the prolonged amenorrhea/ infertility experienced by our patient following TBI, based on the following data: 1) the neuroradiological evidence of pituitary damage (decreased gland size and thickness of the pituitary stalk, this latter abnormality possibly contributing to the hyperprolactinaemia);2) the occurrence of diabetes insipidus (which was transient, but still indicative of some form of lesion of the hypothalamus/stalk/posterior pituitary unit); 3) the presence of isolated failure of gonadotropins, as isolated anterior pituitary hormonal deficiencies are not uncommon post-TBI; 4) the very long duration of amenorrhea, which contrasted with the typically shorter duration of amenorrhea in most women with functional hypothalamic insults, who resume their menses within eight years from a given accident-related stress (range 7-9 yrs); ${ }^{18}$ 5) the lack of any psychological stress following the accident, as the patient resumed her daily activities normally and never consulted psychologists/neurologists or took anti-depressive drugs or sedatives. Even if the empty sella per se is considered an incidental finding, its association with decreased size of the pituitary gland and thinning of the stalk supported the possibility of pituitary damage. Moreover, it is unlikely that hypogonadism and amenorrhoea in this patient were merely a consequence of hyperprolactinemia, 
since PRL was moderately high and responsive to TRH stimulus and clinical galactorrhoea was always absent, thus suggesting a mild, functional alteration of PRL regulation.

Although post-traumatic diabetes insipidus is known to be frequently transient (and this was the case in our patient), spontaneous recovery from established anterior pituitary hormone deficiency is thought to be a very rare event. Our group has reported a case of post-head trauma hypopituitarism (in the form of isolated hypogonadotropic hypogonadism) in a young, infertile man. ${ }^{19}$ Central hypogonadism spontaneously disappeared 11 years after head trauma and, in fact, the patient fathered a baby. ${ }^{18}$ In the present communication we present a second similar case, but this time concerning a young woman in whom spontaneous recovery of ovulation occurred 12 years after the initial insult. A similar phenomenon of late recovery of gonadotropic function has been reported even in genetically based defects, such as Kallman syndrome. ${ }^{20}$

The present case, as well as the few similar reports in men in the literature, ${ }^{19,21}$ demonstrate that spontaneous resolution of a post-traumatic anterior hypopituitarism could be due to pituitary revascularization and cellular pituicyte repopulation. ${ }^{1}$ The present is, therefore, the first report of spontaneous recovery of long-term post-TBI central hypogonadism in a woman.

Post-head trauma hypopituitarism is an increasingly recognized complication of TBI and may interfere significantly with recovery in survivors. ${ }^{2,6}$ Accordingly, we recommend periodic evaluation of the pituitary function in TBI patients with pituitary dysfunction in order to identify potential recovery and avoid unnecessary and expensive therapies, some of which are implemented on a lifelong basis. Another reason for our recommendation is the medicolegal implication of a very important issue, i.e. fertility. For instance, a patient could obtain monetary compensation for claimed damages (e.g. infertility), which, in subsequent months or years, might resolve spontaneously.

\section{REFERENCES.}

1. Benvenga S, Campenní A, Ruggeri RM, Trimarchi F, 2000 Hypopituitarism secondary to head trauma. J Clin
Endocrinol Metab 85: 1353-1361.

2. Schneider HJ, Aimaretti G, Kreitschmann-Andermahr I, Stalla GK, Ghigo E, 2007 Hypopituitarism. Lancet 369: 1461-1470.

3. Agha A, Rogers B, Sherlock M, et al, 2004 Anterior pituitary dysfunction in survivors of traumatic brain injury. J Clin Endocrinol Metab 89: 4929-4936.

4. Kreitschmann-Andermahr I, Hoff C, Saller B, et al, 2004 Prevalence of pituitary deficiency in patients after aneurysmal subarachnoid hemorrhage. J Clin Endocrinol Metab 89: 4986-4992.

5. Benvenga S, 2005 Brain injury and hypopituitarism: the historical background. Pituitary 8: 193-195.

6. Schneider HJ, Kreitschmann-Andermahr I, Ghigo E, Stalla GK, Agha A, 2007 Hypothalamo-pituitary dysfunction following traumatic brain injury and aneurysmal subarachnoid hemorrhage: a systematic review. JAMA 298: 1429-1438.

7. Aimaretti G, Ambrosio MR, Di Somma C, et al, 2005 Residual pituitary function after brain injury-induced hypopituitarism: a prospective 12-month study. J Clin Endocrinol Metab 90: 6085-6092.

8. Herrmann BL, Rehder J, Kahlke S, et al, 2006 Hypopituitarism following severe traumatic brain injury. Exp Clin Endocrinol Diabetes 114: 316-321.

9. Berg C, Oeffner A, Schumm-Draeger PM, et al, 2009 Prevalence of Anterior Pituitary Dysfunction in Patients following Traumatic Brain Injury in a German Multi-centre Screening Program. Exp Clin Endocrinol Diabetes 118: 139-144.

10. Klose M, Juul A, Struck J, Morgenthaler NG, Kosteljanetz M, Feldt-Rasmussen U, 2007 Acute and longterm pituitary insufficiency in traumatic brain injury: a prospective single-centre study. Clin Endocrinol (Oxf) 67: 598-606.

11. Tanriverdi F, Senyurek H, Unluhizarci K, Selcuklu A, Casanueva FF, Kelestimur F, 2006 High risk of hypopituitarism after traumatic brain injury: a prospective investigation of anterior pituitary function in the acute phase and at 12-months after the trauma. J Clin Endocrinol Metab 91: 2105- 2111.

12. Schneider HJ, Schneider M, Saller B, et al, 2006 Prevalence of anterior pituitary insufficiency 3 and 12 months after traumatic brain injury. Eur J Endocrinol 154: 259265.

13. Sesmilo G, Halperin I, Puig-Domingo M, 2007 Endocrine evaluation of patients after brain injury: what else is needed to define specific clinical recommendations? Hormones (Athens) 6: 132-137.

14. Agha A, Rogers B, Mylotte D, et al, 2004 Neuroendocrine dysfunction in the acute phase of traumatic brain injury. Clin Endocrinol (Oxf) 60: 584-591

15. Lee SC, Zasler ND, Kreutzer JS, 1994 Male pituitarygonadal dysfunction following severe traumatic brain injury. Brain Inj 8: 571-577.

16. Woolf PD, Hamill RW, McDonald JV, Lee LA, Kelly 
M, 1986 Transient hypogonadotrophic hypogonadism after head trauma: effects on steroid precursors and correlation with sympathetic nervous system activity. Clin Endocrinol (Oxf) 25: 265-274.

17. Speroff L, Glass R, Kase N 2005. In Clinical Gynecologic Endocrinology and Infertility. 7th edn. Lippincott Williams \& Wilkins, Baltimore, pp, 438-439.

18. Falsetti L, Gambera A, Barbetti L, Specchia CM, 2002 Long term follow-up of functional hypothalamic amenorrhea and prognostic factors. J Clin Endocrinol Metab 87: 500-505.
19. Benvenga S, Lo Giudice F, Campenni A, Longo M, Trimarchi F, 1997 Post-traumatic selective hypogonadotropic hypogonadism. J Endocrinol Invest 20: 675-680.

20. Raivio T, Falardeau J, Dwyer A, et al, 2007 Reversal of idiopathic hypogonadotropic hypogonadism. N Engl J Med 357: 863-873.

21. Agha A, Ryan J, Sherlock M, Thompson CJ, 2005 Spontaneous recovery from posttraumatic hypopituitarism. Am J Phys Med Rehabil 84: 381-385. 